\title{
Oligodendroglioma metastasis to the bone marrow mimicking multiple myeloma: A case report
}

\author{
YUAN JIAN $^{1 *}$, WEN GAO $^{1 *}$, YIN WU $^{1}$, YANCHEN LI $^{1}$, YONG ZHANG $^{2}$, \\ GUANGZHONG YANG $^{1}$ and WENMING CHEN ${ }^{1}$ \\ ${ }^{1}$ Department of Hematology, Beijing Chaoyang Hospital, Capital Medical University, Beijing 100020;
${ }^{2}$ Department of Hematology, Beijing Luhe Hospital, Capital Medical University, Beijing 101100, P.R. China
}

Received March 13, 2015; Accepted April 8, 2016

DOI: $10.3892 / 01.2016 .4568$

\begin{abstract}
The present study reports a case of a 59-year-old male suffering from oligodendroglioma that metastasized to the bone marrow (BM). The metastasis was detected 5 years after craniotomy was performed for the resection of the primary tumor; however, it manifested as multiple myeloma (MM)-like bone lesions, a small $\mathrm{M}$ component and myeloma cell-like morphology in the BM. A brain magnetic resonance imaging scan was performed; evidence from the previously performed oligodendroglioma resection was observed on the scan, but there were no significant findings, which made the diagnosis particularly challenging. The patient exhibited no response to the multiple combination therapies administered targeting MM and oligodendroglioma, and subsequently developed epilepsy and pneumonia, prior to succumbing to multiple organ failure. Among the various tumor types involving the central nervous system, oligodendroglioma is the least likely to metastasize; thus, distant metastases from brain oligodendrogliomas are extremely rare. To the best of our knowledge, this is the first case of metastatic oligodendroglioma presenting with typical MM-like symptoms and without any recurrence in the brain.
\end{abstract}

\section{Introduction}

Multiple myeloma (MM) is a clonal plasma cell malignancy associated with bone, renal, hematological and neurological complications (1). Among the most common symptoms of MM are ostealgia and multiple bone lesions, which are also symptoms of bone marrow (BM) metastatic tumors (2). Since these two diseases are highly similar with regard to susceptible

Correspondence to: Professor Wenming Chen, Department of Hematology, Beijing Chaoyang Hospital, Capital Medical University, 8 Gongti South Road, Beijing 100020, P.R. China

E-mail: xybxx@ccmu.edu.cn

*Contributed equally

Key words: oligodendroglioma, bone marrow metastasis, multiple myeloma, M component, bone lesions populations, clinical features and BM cell morphology, it is often difficult to differentiate between them in cases when the primary lesion is unclear.

Oligodendroglioma is a rare type of tumor accounting for $\sim 5 \%$ of all primary brain tumors (3). Oligodendroglioma predominantly occurs in adults, with peak incidence occurring in patients between the fourth and sixth decades of life (4). Among the various types of tumor involving the central nervous system (CNS), oligodendroglioma is the least likely to metastasize $(5,6)$. Metastasis of oligodendroglioma to the $\mathrm{BM}$ is even more rare, and only a few cases have been reported to date (7-11). The present study reports a case of oligodendroglioma, in which metastasis to the BM was detected 5 years after craniotomy was performed for the resection of the primary tumor; however, the metastasis manifested as MM-like bone lesions, a small M component and myeloma cell-like morphology in the BM, which made the diagnosis particularly challenging. Written informed consent was obtained from the patient's family for publication of this study.

\section{Case report}

A 59-year-old male patient with chronic hepatitis B presented to Beijing Chaoyang Hospital (Beijing, China) on January 20, 2014, with lower back pain that had persisted for 6 months and multiple subcutaneous masses that had been apparent for 1 month. The patient had a history of intracranial oligodendroglioma, which had been treated with craniotomy and a gross total resection 5 years prior to admission. At 6 months prior to the current admission, the patient presented to another hospital with lower back pain accompanied by reduced activity after strenuous exercise. A lumbar magnetic resonance imaging (MRI) scan (Signa HDxt 3.0T scanner; GE Healthcare Bio-Sciences, Pittsburgh, PA, USA) was performed in a different hospital, revealing a compression fracture of the T12 vertebra. Subsequently, BM aspiration and biopsy were scheduled. The aspiration detected the presence of $85.5 \%$ myeloma-like cells in the BM, most of which tended to be myeloblasts, while mitoses and degenerated tumor cells could also be observed, according to the cell morphology. The biopsy indicated hyperplastic BM proliferation with scattered infiltration of lymphocytes and plasmacytes. The streptavidin-peroxidase method was employed in paraffin sections for immunohistochemical staining (all 
A

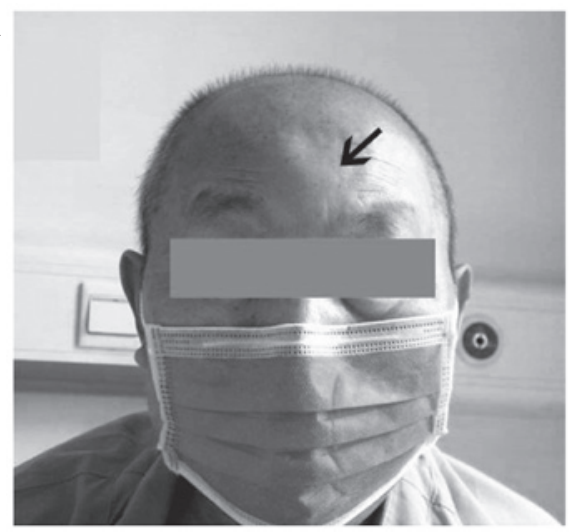

B

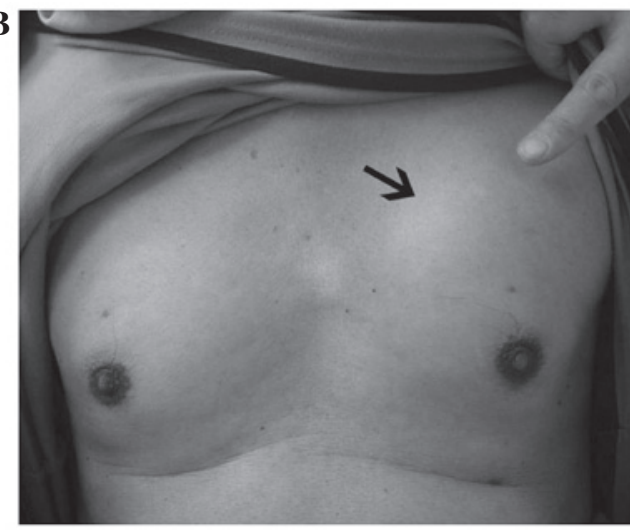

Figure 1. Subcutaneous masses (arrows) on the patient's (A) forehead and (B) left side of the chest.

antibodies used were working solutions with no requirement for dilution, and were mouse anti-human monoclonal, obtained from Fuzhou Maixin Biotech. Co., Ltd., Fujian, China, unless otherwise stated), which revealed clustered and scattered CD138 positivity (catalog no. MAB-0200), minimal CD20 (catalog no. TA800385; dilution, 1:150; OriGene Technologies, Rockville, MD, USA), $15 \%$ CD235a positivity (catalog no. MAB-0603) and CD3 positivity (catalog no. RB-9039), scattered CD38 positivity (catalog no. MAB-0341), negative CD56 reactivity (catalog no. ZM-0057; Zhongshan Golden Bridge Biotechnology Co., Ltd., Beijing, China) and 50\% myeloperoxidase (rabbit anti-human polyclonal; catalog no. RAB-0379) positivity. Flow cytometric, chromosome and fluorescence in situ hybridization (FISH) analyses were not performed, as the BM aspiration was a dry tap at that time. Due to the patient's history of oligodendroglioma, a brain MRI scan was performed; evidence from the previous oligodendroglioma resection was observed on the scan, but there were no significant findings. Serum immunofixation electrophoresis revealed a small $\mathrm{M}$ component of immunoglobulin $\mathrm{G}(\mathrm{IgG})-\kappa$. IgG concentration was $14.3 \mathrm{~g} / \mathrm{l}$ (normal range, 7.5-15.6 g/l), and urine Bence-Jones protein was negative, while peripheral blood leukocyte $\left(4.87 \times 10^{9} / 1\right)$ and platelet $\left(144 \times 10^{9} / 1\right)$ counts, and hemoglobin concentration $(138 \mathrm{~g} / \mathrm{l})$ were within their normal ranges (4-10x10 $/ 1,100-300 \times 10^{9} / 1$ and $120-160 \mathrm{~g} / 1$, respectively). Serum calcium and creatinine levels were normal, while serum alkaline phosphatase (ALP) was markedly elevated (803 IU/l; normal range, 40-160 U/l).

Based on these findings, the patient was diagnosed with MM IgG- $\kappa$ subtype at Durie-Salmon stage III according to the diagnostic criteria defined by the International Myeloma Working Group (12), and subsequently received 5 cycles of melphalan + prednisolone + thalidomide (MPT) combination therapy (6 mg melphalan on days 1-7; $60 \mathrm{mg}$ prednisolone on days 1-7; and $200 \mathrm{mg}$ /day thalidomide, every day, for a 28-day cycle), followed by 1 cycle of melphalan + prednisolone + cyclophosphamide combination therapy (6 mg melphalan on days 1-7; $60 \mathrm{mg}$ prednisolone on days 1-7; $0.2 \mathrm{~g}$ cyclophosphamide on day 1 and $1.0 \mathrm{~g}$ on day 4; and $200 \mathrm{mg} /$ day thalidomide, every day, for a 28-day cycle). Prior to the third cycle of MPT therapy, an additional BM aspiration was performed, in order to evaluate the efficiency of the treatment. The BM aspirate was diluted, which revealed that the BM proliferation was markedly reduced, and the 'myeloma cells' were rarely observable. A BM biopsy revealed a few scattered plasma cells, which were partially positive for CD138, and $\kappa$ (catalog no. MAB-0356) and $\lambda$ (catalog no. MAB-0357) light chains following immumohistochemical staining. Flow cytometry did not detect any abnormal plasma cells. Quantitative polymerase chain reaction showed that the expression of $M Y C$ was slightly elevated (21.33\%) compared with ABL proto-oncogene 1. In addition, prior to the administration of the third cycle of MPT therapy, the patient suddenly presented with aconuresis, logagnosia and paralysis of the right limbs. A brain MRI scan was performed, which indicated cerebral infarction. Furthermore, the location of the oligodendroglioma resection showed no obvious changes, while the areas of skull destruction had clearly increased and expanded compared with the MRI scan performed 2 months prior. These symptoms were relieved following the administration of anticoagulants and antiplatelet drugs.

After the 6 cycles of two different combination therapies, the patient's lower back pain was relatively relieved; however, he gradually developed multiple painless and immovable subcutaneous masses on his forehead and the left side of his chest (Fig. 1). The patient was admitted Beijing Chaoyang Hospital for further diagnosis and treatment. Upon the current admission, key laboratory tests were repeated. BM aspiration detected the presence of $43 \%$ 'immature plasma cells' (Fig. 2), and the BM biopsy yielded mostly cortical bone and did not reveal any hemopoietic tissue. As the BM aspiration was again a dry tap, flow cytometric, chromosome and FISH analyses could not be performed. The serum immunofixation electrophoresis showed no $\mathrm{M}$ component or Bence-Jones protein, and the concentration of IgG was $8.46 \mathrm{~g} / \mathrm{l}$. Chest X-ray (Gendex-Del ATC525 X-ray Generator; Del Medical Inc., Harrison, NY, USA) showed a diffusely increased bone density and multiple bone lesions on the ribs and vertebrae. Several soft tissue masses were visible on the scan. On MRI of the thoracic spine, heterogeneous signals were detected in the thoracic vertebrae, and a soft tissue mass was found on the left 7 th rib (Fig. 3). In addition, the patient was diagnosed with moderate anemia (hemoglobin, $88 \mathrm{~g} / \mathrm{l}$ ) upon admission. Serum calcium and creatinine were normal, while the ALP levels remained elevated (473 IU/1). Neuron-specific enolase was also found to be increased at $60.67 \mathrm{ng} / \mathrm{ml}$ (normal range, $0-16.3 \mathrm{ng} / \mathrm{ml}$ ), while $\beta 2$ microglobulin was normal (2.49 mg/l; normal range, $1.09-2.53 \mathrm{mg} / \mathrm{l})$. 


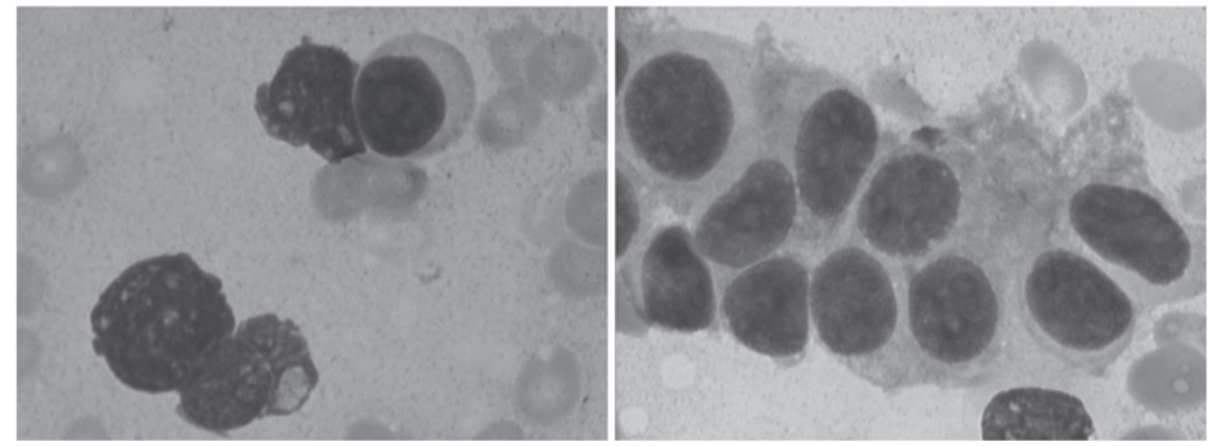

Figure 2. Morphology of the abnormal cells found in the bone marrow aspirate highly resembled that of myeloma cells. The cells varied in size; the majority of them were scattered, while some were clustered. Most abnormal cells were large and round or oval-shaped. The cytoplasm was little to moderate. The nuclei were large and round or oval-shaped with thin chromatin fibers. Binuclear and multinuclear cells were observed in the smear. Hematoxylin and eosin staining; magnification, $\mathrm{x} 1,000$.
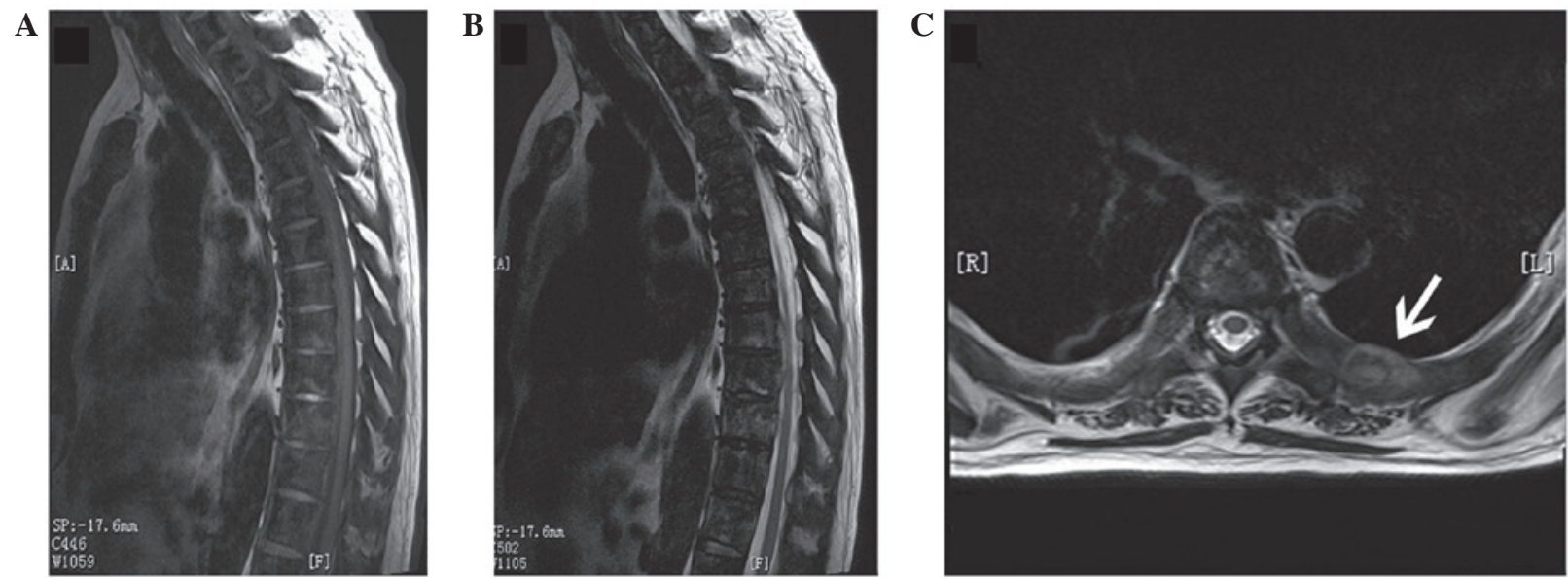

Figure 3. MRI of the thoracic spine. (A) T1- and (B) T2-weighted MRI revealed heterogeneous signals in the thoracic vertebrae; (C) axial T2-weighted MRI revealed a soft tissue mass on the left 7 th rib. MRI, magnetic resonance imaging.
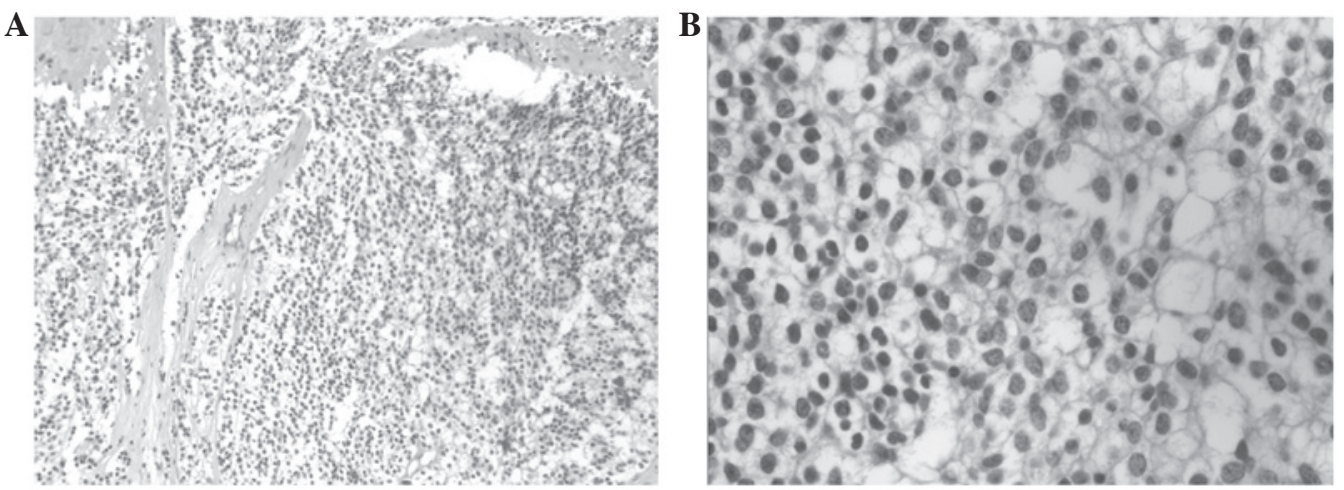

Figure 4. Biopsy of a subcutaneous mass found in left side of the chest. (A) Low-power microscopy (H\&E staining; magnification, x100) showed fibrous tissue with tumor cell infiltration, while (B) high-power (H\&E staining; magnification, $\mathrm{x} 400$ ) revealed that these cells had a consistent size and a transparent cytoplasm. H\&E, hematoxylin and eosin.

Based on the aforementioned findings, the patient was diagnosed with refractory non-secretory MM, without excluding extramedullary plasmocytoma. Subsequently, 1 cycle of cyclophosphamide + thalidomide + daunorubicin + dexamethasone combination therapy (1.2 $\mathrm{g}$ cyclophosphamide on day $1 ; 100 \mathrm{mg} / \mathrm{day}$ thalidomide, every day; $20 \mathrm{mg}$ daunorubicin on days 1-4; and $20 \mathrm{mg}$ dexamethasone on days 1-4, for a 28-day cycle) was administered, followed by 1 cycle of cisplatin + etoposide + cyclophosphamide + dexamethasone + thalidomide (15 mg cisplatin on days 1-4; $60 \mathrm{mg}$ etoposide on days 1-4; $0.4 \mathrm{~g}$ cyclophosphamide on days 1-4; $20 \mathrm{mg}$ dexamethasone on days 1-4; and $100 \mathrm{mg}$ /day thalidomide, every day, for a 28-day cycle). After these 2 courses, the symptoms of subcutaneous masses and anemia showed no improvement. At this time, a biopsy of the subcutaneous mass found in the left side of the chest was performed. Microscopy showed 

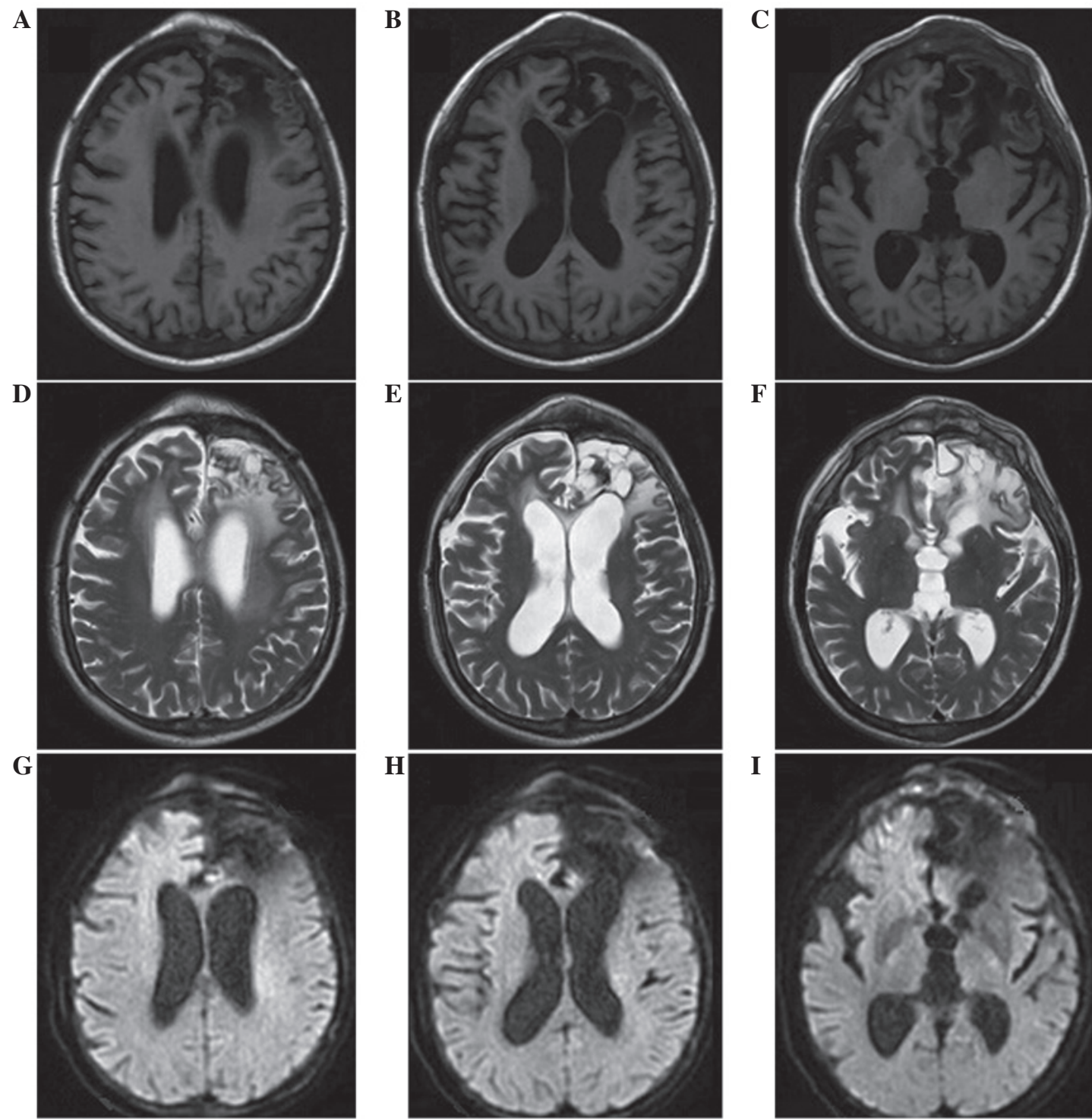

Figure 5. Brain MRI revealed evidence of prior oligodendroglioma resection without current observable primary malignant tumors. (A-C) T1-weighted MRI; (D-F) T2-weighted MRI; (G-I) diffusion-weighted imaging at various section levels. MRI, magnetic resonance imaging.

fibrous tissue with tumor cell infiltration; cells were consistent in size and had a transparent cytoplasm (Fig. 4). Immunohistochemical staining showed the following characteristics: Negative reactivity for vimentin (catalog no. TA801297; dilution, 1:150; OriGene Technologies), cytokeratin (CK; catalog no. MAB-0671), H-CK (catalog no. Kit-0020), CK-L (catalog no. MAB-0051), CD38 (catalog no. MAB-0341), CD138 (catalog no. MAB-0200), $\kappa$ (catalog no. MAB-0356), $\lambda$ (catalog no. MAB-0357), multiple myeloma oncogene 1 (catalog no., ZA-0853; Zhongshan Golden Bridge Biotechnology Co., Ltd.), human melanoma black 45 (catalog no. ab787; Abcam, Cambridge, UK), epithelial membrane antigen (catalog no. Kit-0011), synaptophysin (catalog no. RM-9111), chromogranin A (catalog no. MAB-0202), glial fibrillary acidic protein (catalog no. ZM-0118; Zhongshan Golden Bridge Biotechnology Co., Ltd.) and calcitonin (catalog no. ZA-0578; Zhongshan Golden Bridge Biotechnology Co., Ltd.), and posiivity for CD56 (catalog no.ZM-0057; Zhongshan Golden Bridge Biotechnology Co., Ltd.) and S-100 (catalog no. MAB-0697), with a Ki-67 labeling index (catalog no. RB-9043) of <5\%.
These results were consistent with the brain biopsy results of 5 years prior, and the brain MRI scan showed no primary malignant tumors (Fig. 5); therefore, the subcutaneous masses were finally diagnosed as a metastases from oligodendroglioma. The patient was prescribed 2 cycles of temozolomide chemotherapy (200 $\mathrm{mg}$ on day 1 and $250 \mathrm{mg}$ on days $2-5$ of a 28-day cycle). Despite the slight shrinking of the subcutaneous masses, the patient developed epilepsy and pneumonia and eventually succumbed to multiple organ failure on September 13, 2014.

\section{Discussion}

In cases like the present one, making a correct diagnosis is particularly challenging; the symptoms, signs and laboratory test results of this patient met the 2001 World Health Organization diagnostic criteria for MM (13); however, there were certain reasons to question this diagnosis. Firstly, the abnormal cells found in the BM had not been confirmed to be malignant monoclonal plasma cells; their morphology was 
the only indication. Secondly, the patient had no response to the multiple therapies administered, which were specific to MM and extramedullary plasmocytoma. Thirdly, this patient had different bone imaging characteristics from the typical characteristics of MM bone lesions, which usually demonstrate osteoporosis instead of diffusely increased bone density. Finally, the patient's levels of ALP were found to be markedly elevated, which usually indicates osteogenic activity and is rarely observed in MM.

The main reason for the misdiagnosis of the present case is that the key qualitative diagnosis was based only on morphology, as BM aspiration failed to obtain a sufficient sample quantity for flow cytometric, chromosome and FISH analyses. As the morphology of the oligodendroglioma cells in the $\mathrm{BM}$ of the present patient resembled that of MM cells to a considerable extent, and the symptoms and imaging characteristics of the bone lesions were identical to those of MM bone lesions, the aforementioned evidence indicating a diagnosis other than MM, which could have prevented the misdiagnosis, were ignored upon the patient's first admission. In addition, despite the patient's history of oligodendroglioma, the primary tumor did not show any signs of recurrence, which contributed to the misdiagnosis.

Distant metastasis from brain oligodendroglioma is considered rare (14-16). The presence of the blood-brain barrier, the absence of lymphatics within the CNS, the short survival time of these patients, the inaccessibility of the venous system to the neoplastic cells, the host's immune response and possibly certain biological features of the transformed cells are believed to be the main barriers to the spread of gliomas outside the CNS (15).

The present case exhibited certain considerably rare features, such as the long disease-free interval, the length of time that the patient survived, and the presence of BM metastasis without any primary tumor recurrence in the brain. This metastasis may have been caused by cellular diffusion through the meningeal venous system following surgery, and tumor cell dormancy may explain why the patient remained asymptomatic for such a long period of time without a residual tumor (11).

According to the literature and to the best of our knowledge, this the first report of metastatic oligodendroglioma presenting with myeloma-like symptoms without any tumor recurrence in the brain. In summary, extracranial metastases of oligodendroglioma can occur; however, they are extremely rare. In the present and previously reported cases (7-11), bone metastases occurred following prior craniotomy. In such cases, the BM should be carefully evaluated for glial immunohistochemical markers and using FISH analysis. In addition, the current case highlights the importance of confirming the monoclonality of plasma cells prior to making a final diagnosis of $\mathrm{MM}$, in order to facilitate differentiation between BM metastatic tumors and MM.

\section{References}

1. Palumbo A and Anderson K: Multiple myeloma. N Engl J Med 364: 1046-1060, 2011.

2. Roodman GD: Mechanisms of bone metastasis. New Engl J Med 350: 1655-1664, 2004

3. Leonardi MA and Lumenta CB: Oligodendrogliomas in the CT/MR-era. Acta Neurochir (Wien) 143: 1195-1203, 2001.

4. Van den Bent MJ, Reni M, Gatta G and Vecht C: Oligodendroglioma. Crit Rev Oncol Hematol 66: 262-272, 2008.

5. Smith DR, Hardman JM and Earle KM: Metastasizing neuroectodermal tumors of the central nervous system. J Neurosurg 31: 50-58, 1969.

6. Sha SJ, Wu HP, Lu K, Chen HJ, Huang PH, Huang SH and Hsu CT: Extraneural metastases of anaplastic oligodendroglioma. APMIS 122: 660-662, 2014.

7. Al-Ali F, Hendon AJ, Liepman MK, Wisniewski JL, Krinock MJ and Beckman K: Oligodendroglioma metastatic to bone marrow. AJNR Am J Neuroradiol 26: 2410-2414, 2005.

8. Gru AA, Fulling K and Perry A: A 39 year-old man with a cerebellar mass and pancytopenia. Brain Pathol 22: 251-254, 2012.

9. Li G, Zhang Z, Zhang J, Jin T, Liang H, Gong L, Cui G, Yang H, He S, Zhang Y and Gao G: Occipital anaplastic oligodendroglioma with multiple organ metastases after a short clinical course: A case report and literature review. Diagn Pathol 9: 17, 2014.

10. Cordiano V, Miserocchi F and Storti M: Bone marrow metastases from anaplastic oligodendroglioma presenting with pancytopenia and hypogammaglobulinemia: A case report. Tumori 97: 808-811, 2011.

11. Tanaka Y, Nobusawa S, Ikota H, Yokoo H, Hirato J, Ito H, Saito T, Ogura $\mathrm{H}$ and Nakazato Y: Leukemia-like onset of bone marrow metastasis from anaplastic oligodendroglioma after 17 years of dormancy: An autopsy case report. Brain Tumor Pathol 31: 131-136, 2014.

12. International Myeloma Working Group: Criteria for the classification of monoclonal gammopathies, multiple myeloma and related disorders: A report of the International Myeloma Working Group. Br J Haematol 121: 749-757, 2003.

13. Jaffe ES, Harris NL and Stein H: World Health Organization Classification of Tumors. IARC Press, Lyon, France, pp142-145, 2001.

14. Maiuri F, Del Basso De Caro ML, Iaconetta G, Peca C, Esposito M and de Divitiis E: Prognostic and survival-related factors in patients with well-differentiated oligodendrogliomas. Zentralbl Neurochir 67: 204-209, 2006.

15. Sharma A, Agarwal A, Sharma MC, Anand M, Agarwal S and Raina V: Bone marrow metastasis in anaplastic oligodendroglioma. Int J Clin Pract 57: 351-352, 2003.

16. Zustovich F, Della Puppa A, Scienza R, Anselmi P, Furlan C and Cartei G: Metastatic oligodendrogliomas: A review of the literature and case report. Acta Neurochir (Wien) 150: 699-703, 2008. 